\title{
COMBINATORIAL EXAMPLES IN UNIVERSAL COALGEBRA. II
}

\author{
ROBERT C. DAVIS
}

\begin{abstract}
Several new categories of graph-like structures are described. These extend and (at least in a sense) complete the list of categories cotripleable over the category of sets for which the right adjoint at 2 has four elements.
\end{abstract}

1. Introduction. Universal coalgebra differs from universal algebra, in that it is fairly easy to describe the categories that are quasi-cotripleable over sets $[\mathbf{1}, \mathbf{5}]$ but not so easy to determine which of these are actually cotripleable.

In an attempt to begin to clarify this problem, we have searched for those cotripleable categories, determined by a single operation $\omega: 2 \rightarrow 2$, for which the value of the right adjoint at a two-element set (denoted by $F(2)$ ) has only four elements. First, we searched for possible values of $F(2)$ by searching for all injective four-element structures with suitable symmetries. Though many were found, all of them proved to be more-or-less-obvious notational variants of exactly seven distinct types. In Table 1, a natural example of each of these types is given, where $A=F(2)=\{1,2,3,4\}$, the adjunction $A \rightarrow\{0,1\}$ sends 1,2 to 0 and 3,4 to 1 , and we have listed $\omega_{A}(E)$ for each subset $E$ of $A$.

\section{TABLE 1}

$\begin{array}{cccccccc}E & \text { I } & \text { II } & \text { III } & \text { IV } & \text { V } & \text { VI } & \text { VII } \\ \varnothing & 14 & \varnothing & \varnothing & A & A & A & A \\ 1 & 14 & 13 & 1 & 14 & A & A & 124 \\ 2 & 124 & \varnothing & 23 & A & A & A & A \\ 3 & 134 & \varnothing & 23 & A & A & A & A \\ 4 & 14 & 24 & 4 & 14 & A & A & 134 \\ 12 & 124 & 13 & 123 & 14 & 14 & 14 & 14 \\ 34 & 134 & 24 & 234 & 14 & 14 & 14 & 14 \\ 13 & 134 & 13 & 123 & 14 & A & 14 & A \\ 24 & 124 & 24 & 234 & 14 & A & 14 & A \\ 14 & 14 & A & 14 & A & A & A & A \\ 23 & A & \varnothing & 23 & A & A & A & A \\ 234 & A & 24 & 234 & 14 & A & A & 124 \\ 134 & 134 & A & A & A & A & A & A \\ 124 & 124 & A & A & A & A & A & A \\ 123 & A & 13 & 123 & 14 & A & A & 134 \\ A & A & A & A & A & A & A & A\end{array}$

Received by the editors January 28, 1983.

1980 Mathematics Subject Classification. Primary 18C15; Secondary 08C05.

Key words and phrases. Cotripleable category, right adjoint. 
Each of these seven is actually $F(2)$ for at least one cotripleable category. The first four are well known; I, II, and III are described in [1] and IV in [4]. In fact, type III corresponds to two different categories, $Z_{2}$-sets and sets equipped with equivalence relations. The reason for this is that a $Z_{2}$-set can be regarded as a set with an equivalence relation whose classes (the orbits) have no more than two elements. Furthermore, any cardinal could be used as an upper bound-e.g., there is the category of sets with equivalence relations whose equivalence classes are all finite. A similar phenomenon will be found for type VI; other instances may well remain to be discovered, so we cannot yet claim that our list of categories is complete. Types V, VI, and VII seem to be new and are described in the remaining sections of this paper. Table 2 summarizes the situation, listing the categories in order of increasing size of $F(3)$. Here, $M$ means the monoid $\{1, m\}$ with $m^{2}=m$.

TABLE 2

\begin{tabular}{llcc} 
Type & Category & $|F(3)|$ & $|F(n)|$ \\
\cline { 1 - 1 } I & Sets over 2 & 6 & $2 n$ \\
II & $M$-sets & 9 & $n^{2}$ \\
IIIA & $Z_{2}$-sets & 9 & $n^{2}$ \\
IIIB & Sets with equivalence relations & 12 & $2^{n-1} n$ \\
IV & Multigraphs (without loops) & 12 & $n\left(1+\frac{1}{2} n(n-1)\right)$ \\
V & Double graphs, 1st kind & 21 & $n\left(n^{3}-4 n^{2}+6 n-2\right)$ \\
VIA & Double graphs, 2nd kind & 21 & $\frac{1}{2} n\left(n^{3}-2 n^{2}+n+2\right)$ \\
VIB & Multiple graphs & 30 & $n\left(1+\frac{1}{2} n(n-1)\left(2^{n-1}-1\right)\right)$ \\
VII & Double graphs, 3rd kind & 39 & $n\left(n^{3}-n^{2}-3 n+4\right)$
\end{tabular}

It should be noted that there are phenomena that begin only at larger values of $|F(2)|$. For example, $\mu$-representations of $M$ (see [2]) - which can be regarded as sets equipped with symmetric, transitive relations-have $|F(2)|=8$; and the category of $M$-sets and open homomorphisms [3] has $|F(2)|=6$.

Also, in every example known to us, whenever $F(2)$ is finite, $F(n)$ is also finite for all $n$. It seems natural to conjecture that this is always the case.

2. Double graphs. We consider a multigraph-like structure, in which an "edge" joining "vertices" $a$ and $b$ consists of $t w o$ distinct "edge-like elements" $x$ and $y$. If $V$ is the set of vertices and $E$ the set of edge-like elements, the underlying set is $V \cup E$. We will call $(a, x, y, b)$ a connecting sequence. Loops and multiple edges are permitted; each element of $E$ belongs to a unique connecting sequence; and if $(a, x, y, b)$ is a connecting sequence then $(a, y, x, b)$ is not, except in type VI.

The simplest way to define a homomorphism would seem to be as a function which preserves each connecting sequence or collapses it to a single vertex. Verification of the dual Beck criterion is then straightforward as in [4], as it is for all examples below. The right adjoint $F(A)$ is easy to construct by setting $V=A$ and noting that for each selection of 2,3 , or 4 elements of $A$, only a finite number of elements need be added to $E$. Thus we obtain a cotripleable category; however, $|F(2)|$ turns out to be 22 . 
We will obtain our examples for V, VIA, and VII by suitably altering this definition of homomorphism so as to allow the endomorphisms of the structures listed in Table 1. This will entail slight changes in the notion of a connecting sequence. Specifically, consider the following functions on $(a, x, y, b)$ and their mirror images:

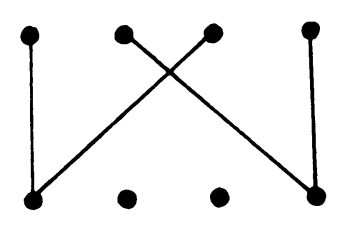

1

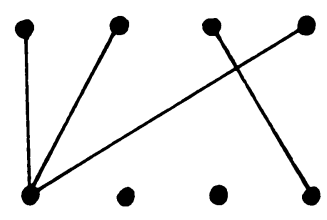

4

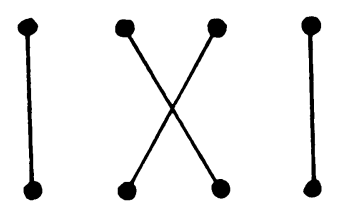

2

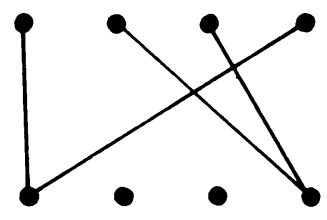

5

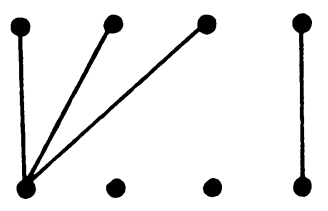

3

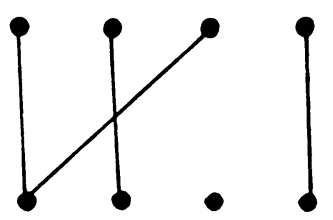

6

Then for type V, we allow \#1,3,4, and 5; for VIA we allow \#2,3,4,5; and for VII, $\# 1,4,5,6$. It is straightforward to verify that these do what we want, and to count the number of elements in $F(n)$. For $V$ this number is $n+2\left(\begin{array}{c}n \\ 2\end{array}\right)+12\left(\begin{array}{c}n \\ 3\end{array}\right)+24\left(\begin{array}{c}n \\ 4\end{array}\right)$; for VIA, where order inside connecting sequences is ignored, it is $n+2\left(\begin{array}{l}n \\ 2\end{array}\right)+12\left(\begin{array}{l}n \\ 3\end{array}\right)+$ $12\left(\begin{array}{l}n \\ 4\end{array}\right)$; and for VII it is $n+2\left(\begin{array}{c}n \\ 2\end{array}\right)+30\left(\begin{array}{c}n \\ 3\end{array}\right)+24\left(\begin{array}{c}n \\ 4\end{array}\right)$.

3. Multiple graphs. Only in case VI have we found an analogue of IIIB. Here we allow an "edge" to be an arbitrary subset $X$ of $E$; again, each element of $E$ belongs to a unique such edge. A homomorphism $f$ may preserve incidence, taking an edge one-to-one onto an edge; it it is not one-to-one, then all elements of the edge are taken to vertices. We also add the condition that if $X$ joins $a$ and $b$, and $f(a) \neq f(b)$, then either $f$ is one-to-one on $X$, or else all of $X$ is taken to one point (vertex). These conditions define a cotripleable category of type VIB with the desired $F(2)$. The number of elements in $F(n)$ is easily seen to be

$$
n+\left(\begin{array}{l}
n \\
2
\end{array}\right)\left(2\left(\begin{array}{l}
n \\
2
\end{array}\right)+3\left(\begin{array}{l}
n \\
3
\end{array}\right)+4\left(\begin{array}{l}
n \\
4
\end{array}\right)+\cdots+n\left(\begin{array}{l}
n \\
n
\end{array}\right)\right) .
$$

As in case III, any cardinal could be used as a bound on the size of $X$-for example, we could require $X$ to be finite-so we have an infinite spectrum of categories of type VI, with VIA and VIB at opposite extremes. 


\section{REFERENCES}

1. R. C. Davis, Universal coalgebra and categories of transition systems, Math. Systems Theory 4 (1970), 91-95.

2. ___ Multivalued operations and universal coalgebra, Proc. Amer. Math. Soc. 32 (1972), 385-388.

3. __ Quasicotripleable categories, Proc. Amer. Math. Soc. 35 (1972), 43-48.

4. Combinatorial examples in universal cobalgebra, Proc. Amer. Math. Soc. 89 (1983), 32-34.

5. J. Rosicky, Considering equational categories (preprint).

Department of Mathematics, Southern Methodist University, Dallas, Texas 75275 